\title{
Developing an app to provide physical and mental health benefits for menopausal women
}

\author{
Prachi Shah, Eleni Stroulia, Sue Ross, Beate Sydora
}

\begin{abstract}
Menopause is the end of a women's menstrual cycle and reproductive years. For some women the transition is without major problems; others struggle with various psychological and physical symptoms. Regular walking exercise can be an easy way to maintain physical and mental health. Previous focus groups revealed that women enjoy and want the social aspect of walking groups. The goal of this study is to test use and effectiveness of a walking app platform-specific for menopausal women that allows them to connect with each other to schedule walks in Edmonton, and track the effect of walking on their symptom changes. Women will be recruited from the Edmonton Menopause clinics and by word of mouth. Interested and consented women receive login information to use the app to schedule and sign-up for walks. Women will complete menopause-specific QOL and symptom questionnaires at their first login and will be prompted to complete them monthly thereafter. Women will also be asked to record their walking durations and intensities. Participating women will be invited to focus groups to discuss their experience using the app. Descriptive statistics will be applied to analyze data collected at the backend from questionnaires and surveys, and qualitative content analysis will be applied to analyze the audio-recorded focus groups. A prototypic online application has been created including a user manual. Access is restricted and all potential participants will be screened after their first login by the research team prior to gaining full access. Women will then be able to use the app features for schedules and surveys. Data collected at the back end by the researchers will determine the use and effectiveness of the app and explore the impact of social walking on menopause symptom changes. This is the first social platform of this kind created specifically for menopausal women. Ultimately we hope that this platform will empower women to seek lifestyle changes such as walking exercises and connect with each other on the communality of menopause. Testing the use and effectiveness of the app will help us to improve and further develop the app.
\end{abstract}

Cite as: Shah P, Stroulia E, Ross S, Sydora B. 2019. Developing an app to provide physical and mental health benefits for menopausal women. Alberta Academic Review, Vol 2 (3) 7, CASCH Special Issue (not peer-reviewed), DOI: 10.29173/aar94.

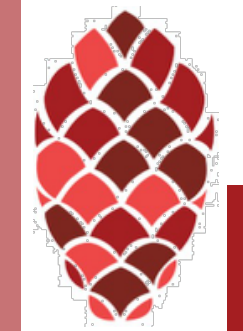

